\title{
Antcin C ameliorates neuronal inflammation due to cerebral haemorrhage by inhibiting the TLR-4 pathway
}

\author{
Wenyuan Ling ${ }^{1,2}$, Ying Cui ${ }^{3}$, Junling Gao ${ }^{4}$, Ran Li ${ }^{5}$, Xiaohua Jiang ${ }^{4}$, Yanxia Tian ${ }^{5}$, Kaijie Wang $^{3}$, Jianzhong Cui ${ }^{1}$ \\ ${ }^{1}$ Department of Surgery, Hebei Medical University, Shijiazhuang, Hebei, China, ${ }^{2}$ Department of Neurosurgery, Weifang People's \\ Hospital, Weifang, China, ${ }^{3}$ Department of Neurosurgery, Tangshan GongrenHospital, Tangshan, Hebei, China, ${ }^{4}$ School of Basic \\ Medical Science, North China University of Science and Technology, Tangshan, Hebei, China, ${ }^{5}$ Hebei Key Laboratory for Chronic \\ Diseases, Tangshan Key Laboratory for Preclinical and Basic Research on Chronic Diseases, Tangshan, China
}

\begin{abstract}
Introduction: This study investigated the protective effects of antcin C against cerebral haemorrhage injury. Material and methods: Cerebral haemorrhage was treated with antcin C $100 \mathrm{mg} / \mathrm{kg}$ i.p. at $60 \mathrm{~min}$ after the induction of cerebral injury. Neurological scores and volumes of cerebral injury were assessed to determine the effects of antcin C, based on oxidative stress and serum mediators of inflammation by ELISA. qRT-PCR was used to estimate the IRNA expression of Toll-like receptor 4 (TLR-4) and interleukin-1 receptor-associated kinase 4 (IRAK4) proteins in the cerebral tissue of rats with cerebral haemorrhage. Western blot assay and histopathology were also performed. Results: The findings suggest that treatment with antcin C reduced the neurological scores and volumes of cerebral injury in cerebral injured rats. Parameters of oxidative stress and cytokine levels were reduced in the serum of the antcin C-treated group compared with the negative control group. Treatment with antcin C ameliorated the expression of TLR-4, IRAK4, and zonula occludens-1 (ZO-1) proteins in the cerebral tissue of cerebral injured rats.

Conclusions: The results revealed that treatment with antcin $C$ protected against cerebral haemorrhage damage by controlling microglia inflammation through the TLR-4 pathway.
\end{abstract}

Key words: antcin C, cerebral haemorrhage, inflammation, oxidative stress, neuronal injury.

\section{Introduction}

Cerebral haemorrhage is a type of stroke resulting from rupture of blood vessels in the brain [16]. Cerebral haemorrhage is one of the major causes of mortality worldwide, occurring in approximately 15 million people every year, with a 30-day mortality rate of $40 \%$ [18]. Secondary injury in cerebral haemorrhage plays an important role in the development of further haemorrhages [3]. Conventional drugs available for the treatment of secondary injury associated with cerebral haemorrhage have several limitations, pointing to the need for the development of new therapeutic agents [1]. Microglia are supporting cells in the central nervous system (CNS) that act as immune cells. The literature suggests that activation of the microglia enhances neuronal degeneration by initiating the apoptosis cascade [17]. Neurodegeneration occurs due to over-activation of microglia by enhancing the production of inflammation mediators such as nitrogen species, reactive oxy-

\section{Communicating author}

Jianzhong Cui, PhD, Department of Surgery, Hebei Medical University, No. 361 Zhongshan East Road, Shijiazhuang, Hebei, 050017, China, e-mail: ClintonLambpbo@yahoo.com 
gen species (ROS), prostaglandin, and cytokine [19]. Neuroinflammation occurs in neurodegeneration due to increased synthesis and release of cytokines, which is regulated by Toll-like receptor 4 (TLR-4) [2]. Moreover, the microglia-associated immune response is linked with TLR-4. Previous research has shown that TLR-4 is involved in lipopolysaccharide (LPS)-induced neuronal injury [13]. Thus, activation of the microglia could be a potential therapeutic target for the management of neurological disorders.

Alternative therapies have shown potential for the management of several disorders, including cerebral injury. Antcin C is a steroid-like compound isolated from Antrodia cinnamomea [20], a mushroom traditionally used as a medicine in China for the management of cancer, hypertension, and liver disorder. Moreover, an extract of $A$. cinnamomea was reported to have neuroprotective effects [5]. Several molecules, including triterpenoids, steroids, and benzenoids, have been isolated from A. cinnamomea [12]. Antcins reportedly improve blood circulation and have anti-inflammatory properties [21]. Several types of antcin compounds are available; antcin $C$ is known for its protective effects against liver injury and oxidative stress by modulating the Nrf2 pathway [10]. Thus, the present report evaluates the protective effects of antcin $C$ against neuroinflammation in a cerebral injured rat model.

\section{Material and methods \\ Animals}

Male Sprague-Dawley rats (180-200 g) and newborn SD rats were maintained under a 12-h light/dark cycle and standard conditions at $24 \pm 3^{\circ} \mathrm{C}$ and relative humidity of $60 \pm 5 \%$. All study protocols were approved by the institutional committee on animal ethics of Hebei Medical University, China (IAEC/HMU/12/2018).

\section{Experimental}

Cerebral haemorrhage was induced in rats by injecting fresh autogenous arterial blood $(50 \mu l)$ into the basal ganglia. All animals were injected with $0.03 \mathrm{ml} / \mathrm{kg}$ of chloral hydrate $(10 \%)$ to produce anaesthesia, followed by isolation of the femoral artery. After $60 \mu$ l of blood was withdrawn, ligation was performed. Animals were placed on the platform of a stereotaxic apparatus, and an incision was made to expose the anterior ridge. Fresh femoral artery blood was injected at a speed of 7-10 $\mu \mathrm{l} / \mathrm{min}$ through a micro-syringe after a hole was created to the right side of the midline and $0.2 \mathrm{~mm}$ in front of the forehead.

Animals were separated into three groups: the sham group, negative control group, and antcin $C$ $100 \mathrm{mg} / \mathrm{kg}$ group. The last group received antcin $C$ $100 \mathrm{mg} / \mathrm{kg}$ i.p. at $60 \mathrm{~min}$ after the induction of cerebral injury. One day after the operation, all animals were sacrificed to assess the effects of antcin $C$ on the cerebral injury.

\section{Neurological score assessment}

Assessment of the neurological score was performed as in a previous study [7]. In brief, six different types of behavioural changes were observed in all animals including climbing, movement of four limbs in symmetry, body proprioception, whisker stimulation, outstretching of the forepaw, and spontaneous activity. A 3-point scale was administered. The neurological score was determined both before and after the induction of cerebral haemorrhage.

\section{Assessment of haemorrhagic injury}

Haemorrhagic injury was determined by estimating the haemorrhagic volume, as in a previous study [9]. Cresyl violet was used to stain the coronal sections at 20 rostral-caudal levels to determine injection volume. Image Pro Plus 6.0 software was used to determine the haemorrhagic volume.

\section{Assessment of cytokines}

Animals were anaesthetised, and blood was drawn from the retro-orbital plexus. Serum was separated using a centrifuge for $10 \mathrm{~min}$ at 2000 RPM. The serum levels of tumor necrosis factor $\alpha$ (TNF- $\alpha$ ), interleukin (IL)-1 $\beta$, and IL-8 were estimated using ELISA kits per the manufacturer's instructions.

\section{Assessment of oxidative stress parameters}

Malondialdehyde (MDA) and ROS were estimated to determine the level of oxidative stress. The MDA level was estimated using a lipid peroxidation MDA assay kit, and the reactive ROS level was detected in the cells using a ROS assay kit.

\section{qRT-PCR}

TRIzol reagent was used to extract the total RNAs, and TaqMan MicroRNA assays were used to 
estimate the mRNAs levels on qRT-PCR. Moloney murine leukaemia virus reverse-transcriptase was used to synthesise complementary DNA by applying $2 \mu \mathrm{g}$ of total RNA to the reverse transcriptase reaction $(20 \mu \mathrm{l})$. The primers listed below were mixed with RT 2 SYBR Green Mastermix to determine gene expression using the Quantitative SYBR Green PCR assay. The relative target gene expression levels were determined using the $2^{-\Delta \Delta C q}$ equation.

\begin{tabular}{ccr}
\hline \multirow{2}{*}{ TLR-4 } & Forward & 5'-AGCTTTGGTCAGTTGGCTCT-3' \\
\cline { 2 - 3 } & Reverse & 5'-CAGGATGACACCATTGAAGC-3' \\
\hline IRAK4 & Forward & 5'-CATCAACTATGGAGTAAGCTGGAC-3' \\
\cline { 2 - 3 } & Reverse & 5'-GTCCAGGGTCGTTTTCTCTG-3' $^{\prime}$ \\
\hline \multirow{2}{*}{-actin } & Forward & 5' $^{\prime}$-GGAGATTACTGCCCTGGCTCCTA-3' \\
\cline { 2 - 3 } & Reverse & 5' $^{\prime}$ - GACTCATCGTACTCCTGCTTGCTG-3' \\
\hline
\end{tabular}

\section{Western blot assay}

Extraction of total protein from cells was performed after treatment with protein lysis buffer. Total protein concentration was estimated using a DC Protein Assay. Isolated protein was separated using a sodium dodecyl sulphate-polyacrylamide gel $(10 \%)$ and filtered with a polyvinylidene difluoride membrane. The membrane was then treated with $5 \%$ fresh non-fat dry milk to block the reaction, incubated at $4^{\circ} \mathrm{C}$ overnight with primary antibodies such as interleukin-1 receptor-associated kinase 4 (IRAK4; 1 : 1000), TLR-4 (1: 500), zonula occludens-1 (ZO-1; $1: 1000$ ), and glyceraldehyde 3-phosphate dehydro-

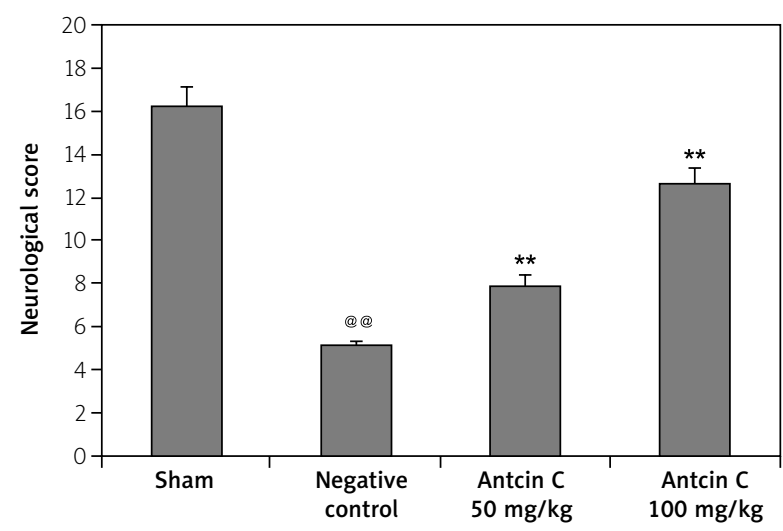

Fig. 1. Antcin $C$ ameliorates the altered neurological score in cerebral haemorrhage injured rats. Mean \pm SEM $(n=10)$, @ $p<0.01$ vs. sham group, ${ }^{* *} p<0.01$ vs. negative control group. genase (GAPDH; $1: 1000)$, and then incubated with secondary antibodies. Densitometric analysis of the blots was performed using Image Lab software.

\section{Histopathological analysis}

Formaldehyde (4\%) was used to fix the brain tissues, which were embedded in paraffin, sectioned to $5-\mu \mathrm{m}$ thicknesses using a microtome, and stained with haematoxylin and eosin (H\&E). A trinocular microscope was used to evaluate pathophysiological changes in the brain tissue.

\section{Statistical analysis}

All data are expressed as the mean \pm standard error of the mean $(n=10)$. One-way analysis of variance was used to perform statistical analyses, with Dunnett's test for post hoc analysis (GraphPad Prism 6.1.; GraphPad Software, Inc., USA). A P-value $<0.05$ was considered significant.

\section{Results}

\section{Antcin C ameliorates the neurological score}

The neurological score was assessed by observing the behaviour of each animal treated with antcin C, as shown in Figure 1. The negative control group exhibited a reduction in the neurological score (behavioural score) relative to the sham-operated group. However, a dose-dependent improvement in the neurological score was observed in the antcin C-treated group compared with the negative control group.

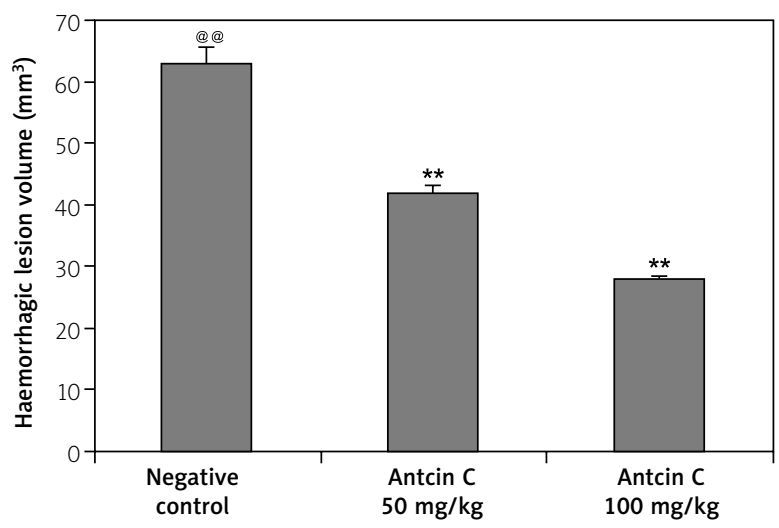

Fig. 2. Antcin $C$ ameliorates the altered volume of haemorrhagic lesions in cerebral haemorrhage injured rats. Mean \pm SEM $(n=10)$, ${ }^{@} p<0.01$ vs. sham group, ${ }^{* *} p<0.01$ vs. negative control group. 
A

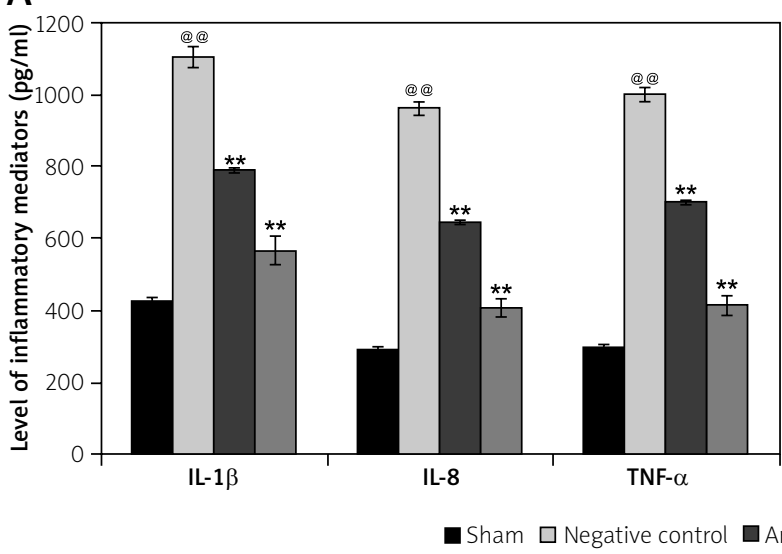

B

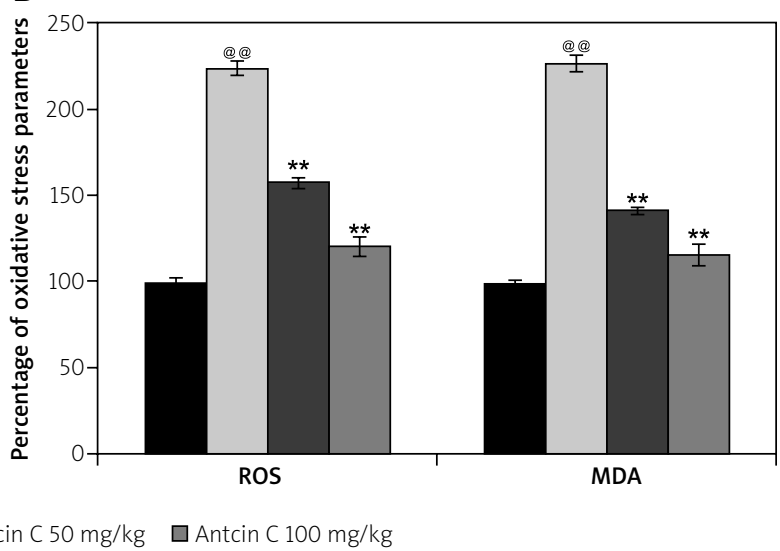

Fig. 3. Antcin $C$ ameliorates the altered level of biochemical parameters in cerebral haemorrhage injured rats. A) Antcin C ameliorates altered level of inflammatory cytokines such as IL-1 $\beta$, IL-8, and TNF- $\alpha$; B) Antcin $C$ ameliorates altered level of oxidative stress parameters such as ROS and MDA. Mean \pm SEM $(n=10)$, @@ $p<0.01$ vs. sham group, ${ }^{\star \star} p<0.01$ vs. negative control group.

\section{Antcin C ameliorates haemorrhagic injury}

Haemorrhagic lesion volume was assessed in antcin C-treated cerebral haemorrhage injured rats. The volume of haemorrhagic lesion in cerebral tissue was as high as $63 \mathrm{~mm}^{3}$ in the negative control group. However, treatment with antcin $C$ reduced the haemorrhagic lesion volume by up to $28 \mathrm{~mm}^{3}$ in cerebral haemorrhage injured rats (Fig. 2).

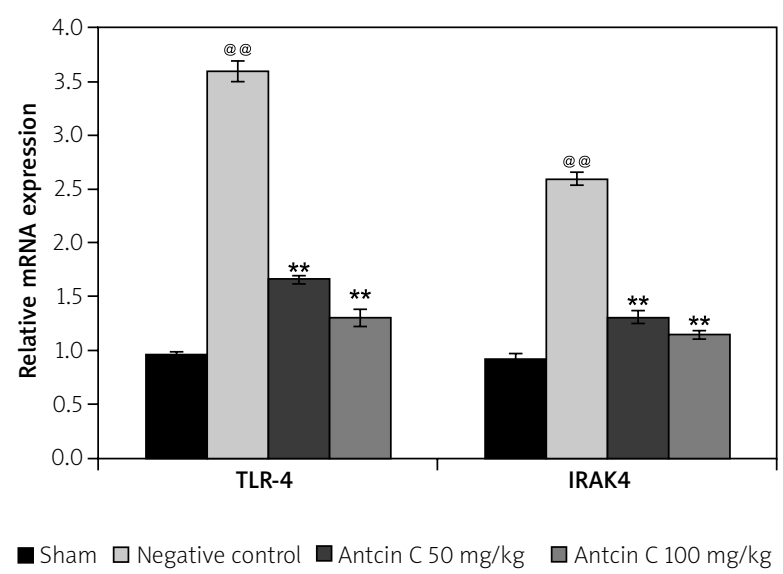

Fig. 4. Antcin $C$ ameliorates altered mRNA expression of TLR-4 and IRAK4 in the cerebral tissues of cerebral haemorrhage injured rats. Mean \pm SEM $(n=10),{ }^{@ @ ~} p<0.01$ vs. sham group, ${ }^{* *} p<0.01$ vs. negative control group.

\section{Antcin C ameliorates inflammatory cytokines and oxidative stress}

Figure 3 presents the biochemical parameter values for the antcin C-treated cerebral haemorrhage injured rats. The levels of cytokines such as $\mathrm{IL}-1 \beta, \mathrm{IL}-8$, and TNF- $\alpha$ were enhanced in the serum of the negative control relative to the sham-operated group. Moreover, the serum cytokine level in the antcin C-treated group was reduced compared with that in the negative control group (Fig. 3A). A change in the oxidative stress level was observed in the antcin C-treated cerebral haemorrhage injured rats, as shown in Figure 3B. The percentages of MDA and ROS production were more enhanced in the serum of the negative control group than in that of the sham-operated group. Moreover, the reduction in the percentage of ROS and MDA was greater in the serum of the antcin $C$ group than in the negative control group.

\section{Antcin $\mathrm{C}$ ameliorates mRNA expression of TLR-4 and IRAK4}

The effects of antcin $C$ on the mRNA expression of TLR-4 and IRAK4 in the cerebral tissues of cerebral haemorrhage injured rats were determined using qRT-PCR. mRNA expression of TLR-4 and IRAK4 was enhanced significantly in the cerebral tissue of the negative control group compared with the sham-operated group. The mRNA expression of TLR-4 and IRAK4 was reduced in the cerebral 

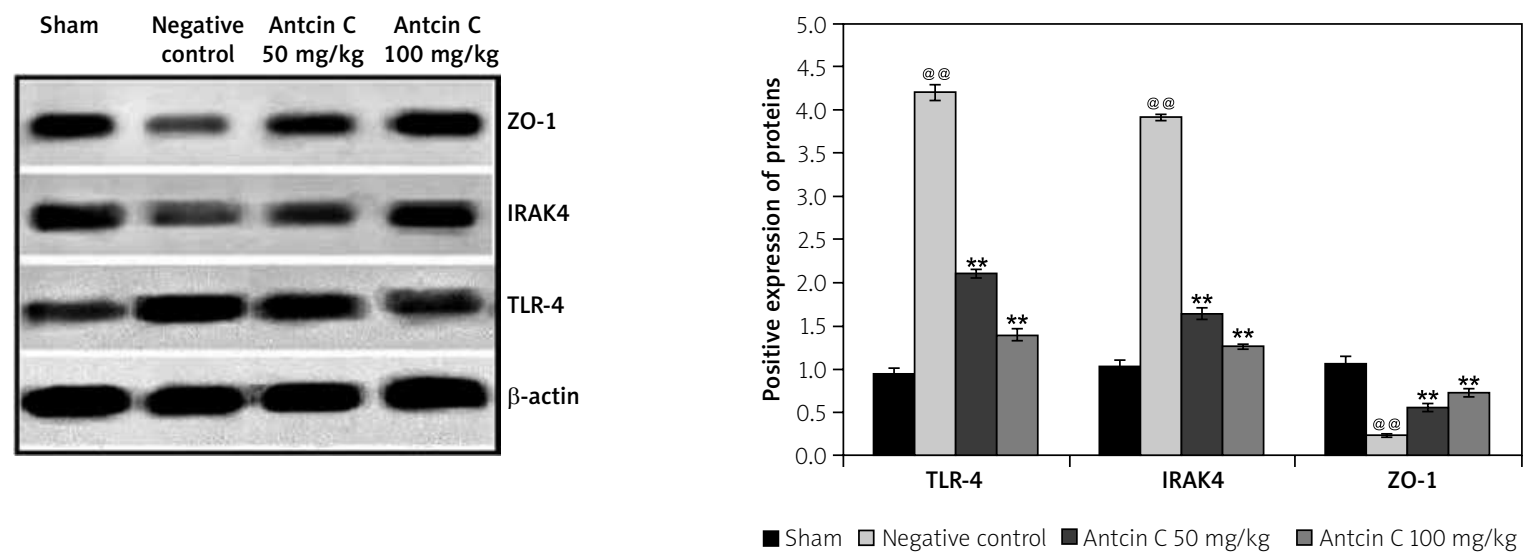

Fig. 5. Antcin $C$ ameliorates the altered expression of ZO-1, TLR-4, and IRAK4 proteins in the cerebral tissues of cerebral haemorrhage injured rats. Mean \pm SEM $(n=10)$, ${ }^{@} p<0.01$ vs. sham group, ${ }^{* *} p<0.01$ vs. negative control group.
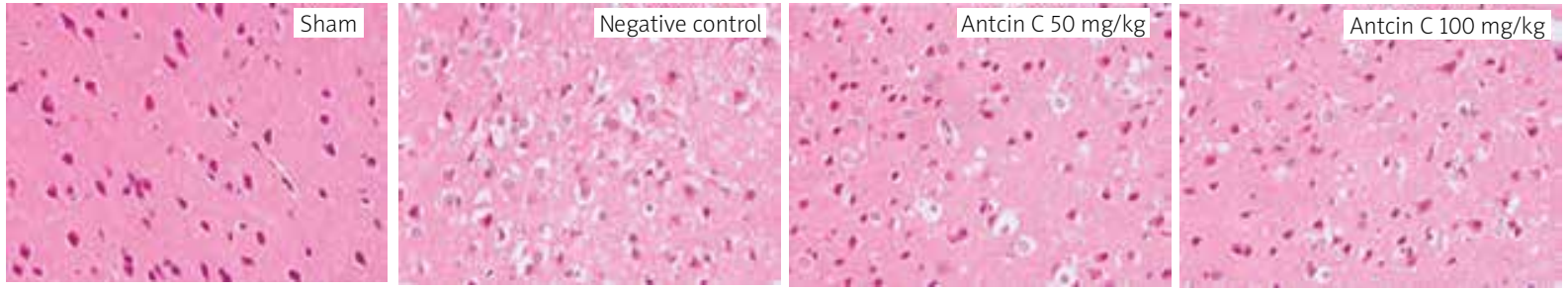

Fig. 6. Antcin $\mathrm{C}$ ameliorates histopathological changes in the cerebral tissues of cerebral haemorrhage injured rats.

tissue of the antcin C-treated group compared with the negative control group (Fig. 4).

\section{Antcin $C$ ameliorates altered expression of TLR-4, IRAK4, and ZO-1 proteins}

The effects of antcin $C$ on the expression of ZO-1, TLR-4, and IRAK4 proteins in the cerebral tissues of cerebral haemorrhage injured rats was estimated using a Western blot assay. The results revealed greater enhancement of the expression of TLR-4 and IRAK4 proteins and reduction in the expression of ZO-1 proteins in the cerebral tissue of the negative control group than in the sham-operated group. Furthermore, treatment with antcin $\mathrm{C}$ ameliorated the altered expression of ZO-1, TLR-4, and IRAK4 proteins in the cerebral tissues of cerebral haemorrhage injured rats (Fig. 5).

\section{Antcin C ameliorates histopathological changes in brain tissue}

Figure 6 presents the histopathological changes in the brain tissue of antcin C-treated cerebral haemorrhage rats by $H \& E$ staining. Brain tissue sections from the sham-operated group appeared normal, whereas the brain tissues of the cerebral haemorrhage group, i.e., the negative control group, exhibited nuclear and cytoplasmic vacuolation in the white matter and cortex. Moreover, the neurocytes were unconsolidated and disordered. However, treatment with antcin C ameliorated these pathological changes in the brain tissues of cerebral haemorrhage injured rats.

\section{Discussion}

Cerebral haemorrhage, a type of stroke that occurs due to blood vessel rupture in the brain, is one of the major causes of mortality throughout the world. Conventional treatment has several limitations, so there is a need for the development of new therapies for the management of cerebral haemorrhage. This study investigated the protective effects of antcin $C$ against cerebral haemorrhage injury. The neurological score and volume of cerebral injury were assessed to determine the effects of antcin C. These effects were estimated by ELISA based on oxidative stress and mediators of inflammation in 
the serum, and qRT-PCR was used to estimate the mRNA expression of TLR-4 and IRAK4 proteins in the cerebral tissue of cerebral haemorrhage injured rats. Western blot assay and histopathology were also performed for the cerebral haemorrhage injured rats.

Previous research has shown that neuronal damage is induced by activation of the inflammatory cascade due to pro-inflammatory molecules [6]. Microglia are supporting cells that play an important role as immune cells of the CNS [14]. In cerebral haemorrhage, activation of the inflammatory pathway occurs due to over-activation of the microglia. Moreover, activation of microglia cells is regulated by several intracellular signalling and transcription factors. Oxidative stress reportedly plays an important role in the activation of the inflammatory cascade [15], and the results of the present study suggest that antcin $C$ treatment reduces the level of oxidative stress and inflammatory mediators in the serum of cerebral injured rats.

The production of cytokines and the NF- $\mathrm{KB}$ signalling pathway are activated through activation of TLR-4, which belongs to the Toll-like receptor family [4]. These changes stimulate innate immunity, and previous studies have revealed that LPS, which is produced by Gram-negative bacteria, activates TLR-4 [19]. The results of the present study suggest that treatment with antcin C significantly reduced the expression of TLR-4 and IRAK 4 proteins in cerebral haemorrhage injured rats. ZO-1, commonly called tight junction protein 1 , alters the integrity of the blood-brain barrier, and it has been observed in cerebral haemorrhage-like pathological conditions [11]. This is involved in further contributions to secondary injury in cerebral haemorrhage. The integrity of the blood-brain barrier is reportedly maintained by enhancing the expression of ZO-1 [8]; data from the present study support this conclusion.

\section{Conclusions}

In conclusion, the results of this investigation reveal that treatment with antcin $C$ protects the cerebellum against cerebral haemorrhage by controlling neuronal injury through the TLR-4 pathway.

\section{Acknowledgements}

All authors of this manuscript are grateful to the Natural Science Foundation of Hebei Province for providing the necessary funding (No. H2019105137) to conduct the work presented here.

\section{Disclosure}

The authors report no conflict of interest.

\section{References}

1. Aguilar MI, Brott TG. Update in intracerebral hemorrhage. Neurohospitalist 2011; 1: 148-159.

2. Azam S, Jakaria M, Kim IS, Kim J, Haque ME, Choi DK. Regulation of Toll-like receptor (TLR) signaling pathway by polyphenols in the treatment of age-linked neurodegenerative diseases: focus on TLR4 signaling. Front Immunol 2019; 10: 1000.

3. Babi MA, James ML. Peri-hemorrhagic edema and secondary hematoma expansion after intracerebral hemorrhage: from benchwork to practical aspects. Front Neurol 2017; 8: 4.

4. Branger J, Knapp S, Weijer S, Leemans JC, Pater JM, Speelman P, Florquin S, van der Poll T. Role of Toll-like receptor 4 in gram-positive and gram-negative pneumonia in mice. Infect Immun 2004; 72: 788-794.

5. Chen CC, Shiao YJ, Lin RD, Shao YY, Lai MN, Lin CC, Ng LT, Kuo YH. Neuroprotective diterpenes from the fruiting body of Antrodia camphorata. J Nat Prod 2006; 69: 689-691.

6. Chen L, Deng H, Cui H, Fang J, Zuo Z, Deng J, Li Y, Wang X, Zhao L. Inflammatory responses and inflammation-associated diseases in organs. Oncotarget 2017; 9: 7204-7218.

7. Clark A, Das JM, Mesfin FB. Trauma Neurological Exam. In: StatPearls [Internet]. StatPearls Publishing, Treasure Island (FL) 2020 (Updated 2020 Mar 1).

8. Daneman R, Prat A. The blood-brain barrier. Cold Spring Harb Perspect Biol 2015; 7: a020412.

9. Falcone GJ, Biffi A, Brouwers HB, Anderson CD, Battey TW, Ayres AM, Vashkevich A, Schwab K, Rost NS, Goldstein JN, Viswanathan A, Greenberg SM, Rosand J. Predictors of hematoma volume in deep and lobar supratentorial intracerebral hemorrhage. JAMA Neurol 2013; 70: 988-994.

10. Gokila Vani M, Kumar KJ, Liao JW, Chien SC, Mau JL, Chiang SS, Lin CC, Kuo YH, Wang SY. Antcin C from Antrodia cinnamomea protects liver cells against free radical-induced oxidative stress and apoptosis in vitro and in vivo through a Nrf2-dependent mechanism. Evid Based Complement Alternat Med 2013; 2013: 296082.

11. Jiao X, He P, Li Y, Fan Z, Si M, Xie Q, Chang X, Huang D. The role of circulating tight junction proteins in evaluating blood brain barrier disruption following Intracranial Hemorrhage. Dis Markers 2015; 2015: 860120.

12. Lee YP, Tsai WC, Ko CJ, Rao YK, Yang CR, Chen DR, Yang MH, Yang CC, Tzeng YM. Anticancer effects of eleven triterpenoids derived from Antrodia camphorata. Anticancer Res 2012; 32: 2727-2734.

13. Lehnardt S, Massillon L, Follett P, Jensen FE, Ratan R, Rosenberg PA, Volpe JJ, Vartanian T. Activation of innate immunity in the CNS triggers neurodegeneration through a Toll-like receptor 4-dependent pathway. Proc Natl Acad Sci USA 2003; 100: 8514-8519.

14. Lenz KM, Nelson LH. Microglia and beyond: innate immune cells as regulators of brain development and behavioral function. Front Immunol 2018; 9: 698. 
15. Mittal M, Siddiqui MR, Tran K, Reddy SP, Malik AB. Reactive oxygen species in inflammation and tissue injury. Antioxid Redox Signal 2014; 20: 1126-1167.

16. Pinho J, Costa AS, Araújo JM, Amorim JM, Ferreira C. Intracerebral hemorrhage outcome: A comprehensive update. J Neurol Sci 2019; 398: 54-66.

17. Podbielska M, Das A, Smith AW, Chauhan A, Ray SK, Inoue J, Azuma M, Nozaki K, Hogan EL, Banik NL. Neuron-microglia interaction induced bi-directional cytotoxicity associated with calpain activation. J Neurochem 2016; 139: 440-455.

18. Qureshi Al, Mendelow AD, Hanley DF. Intracerebral haemorrhage. Lancet 2009; 373: 1632-1644.

19. Tjalkens RB, Popichak KA, Kirkley KA. Inflammatory activation of microglia and astrocytes in manganese neurotoxicity. Adv Neurobiol 2017; 18: 159-181.

20. Wang YJ, Lee SC, Hsu CH, Kuo YH, Yang CC, Lin FJ. Antcins, triterpenoids from Antrodia cinnamomea, as new agonists for peroxisome proliferator-activated receptor $\alpha$. J Food Drug Anal 2019; 27: 295-304

21. Wu YS, Chen SN. Extracted triterpenes from Antrodia cinnamomea reduce the inflammation to promote the wound healing via the STZ inducing hyperglycemia-diabetes mice model. Front Pharmacol 2016; 7: 154. 